\title{
Kasuistik \\ Schwere Intoxikation nach oraler Einnahme von Alkylnitrit („Poppers“)
}

Barbara Bernasconi • Christoph Konrad • Simon Fischer

Ein 23-Jähriger erleidet auf einer Party nach einer versehentlichen oralen Ingestion von Alkylnitriten - sog. „Poppers“ - eine schwere Intoxikation. Das erstmals 1867 als Antianginosum eingesetzte Medikament gewann in den 1960er-Jahren als Partydroge v. a. in homosexuellen Kreisen an Bedeutung. Sie wird wegen ihrer hohen Flüchtigkeit und dem schnellen Wirkungseintritt direkt aus der Flasche inhaliert. Die Wirkdauer bei inhalativer Einnahme ist sehr kurz, hat eine euphorisierende und muskelrelaxierende Wirkung, was zur Vasodilatation und Relaxation des Analsphinkters führt. Bei oraler Ingestion können schwerste Nebenwirkungen in Form einer massiven Methämoglobinbildung und schweren Hypotonien entstehen. Im folgenden Artikel lesen Sie über den Verlauf der potenziell letalen Methämoglobinbildung, ihrer Pathophysiologie und Antidottherapie.

\section{Der Fall}

\section{Präklinische Versorgung $\nabla$}

Einsatz Der Rettungsdienst wird um 23:50 Uhr durch einen Samariter zu einem Partyzelt gerufen mit der Angabe eines somnolenten Patienten mit einer Mischintoxikation, Atmung normal.

Am Einsatzort wartet auf das Team ein 23-jähriger, kaltschweißiger, tiefzyanotischer Patient mit einer Atemfrequenz von 20/min, einer peripheren Sättigung von $88 \%$, einem Blutdruck von $100 / 55 \mathrm{mmHg}$ und einer Sinustachykardie von $135 / \mathrm{min}$. Der GCS beträgt 9 .

Fremdanamnestisch hat der Patient eine unbekannte Menge Alkohol und ein Fläschchen „Poppers“ getrunken. Dem Patienten werden $151 \mathrm{O}_{2}$ via Venturimaske verabreicht, die periphere Sättigung steigt jedoch nicht an. Im Verlauf zeigen sich Blutdruckwerte von 80/40 mmHg. Nach Gabe von $1000 \mathrm{ml}$ Kristalloiden und fraktionierter Gabe von insgesamt $20 \mathrm{mg}$ Ephedrin stabilisieren sich die Blutdruckwerte auf $105 \mathrm{mmHg}$ systolisch und die Herzfrequenz sinkt auf 100/min ab. Mit dem Verdacht auf eine schwere Alkylnitritintoxikation wird der Patient in den Schockraum des Zentrumspitals gebracht.

\section{Versorgung im Schockraum \\ $\nabla$}

Zustand des Patienten Bei der Ankunft im Schockraum sind die Atemwege frei. Der Patient ist tachypnoisch, die Sättigung beträgt mit $151 \mathrm{O}_{2}$ via Venturimaske $66 \%$ bei guter Signalqualität. Es besteht eine Sinustachykardie von 110/min und eine Hypotension von $80 / 30 \mathrm{mmHg}$. Der GCS ist 12, der Patient ist sehr agitiert. Auffallend ist ein tief grau-blaues Haut- und Schleimhautkolorit.

Behandlung Es erfolgt die sofortige problemlose Intubation mit Midazolam, Fentanyl und Rocuronium. Der Patient wird anschließend mit einem inspiratorischen $\mathrm{O}_{2}$-Anteil $\left(\mathrm{FiO}_{2}\right)$ von 1 maschinell beatmet, die Analgosedierung erfolgt mit Isofluran und Fentanyl. Zur Kreislaufstabilisierung bei vasodilatatorischem Schock wird rasch eine kristalloide Lösung über einen großlumigen periphervenösen Zugang gegeben sowie kontinuierlich Noradrenalin. Der Patient wird mit einem arteriellen Katheter, einem zentralvenösen Zugang und einem Blasenkatheter versorgt. Die Blutentnahme über den arteriellen Katheter zeigt

- eine für die Methämoglobinvergiftung typische schokoladenbraune Farbe,

- einen $\mathrm{pH}$ von 7,32,

- einen arteriellen Sauerstoffpartialdruck $\left(\mathrm{PaO}_{2}\right)$ von $38,5 \mathrm{kPa}$ und

- einen Laktatwert von $2,6 \mathrm{mmol} / \mathrm{l}$.

Der Methämoglobinanteil beträgt $43,2 \%$, der Blutalkoholspiegel 1,77\%. In Rücksprache mit dem toxikologischen Zentrum werden $100 \mathrm{mg}$ ( $\mathrm{ca}$. $1,5 \mathrm{mg} / \mathrm{kg} \mathrm{KG}$ ) Methylenblau in einer Kurzinfusion verabreicht, worauf sich der Methämoglobinanteil nach einer halben Stunde auf 22,6\% reduziert. Das Hautkolorit des Patienten wird daraufhin merklich rosiger. Die pulsoxymetrisch gemessene Sättigung beträgt $86 \%$, der arterielle $\mathrm{PaO}_{2}$ $62,8 \mathrm{kPa}$. Der Patient stabilisiert sich soweit, dass er auf die medizinische Intensivstation verlegt werden kann. 


\section{Intensivtherapie}

Weitere Behandlung Die Volumensubstitutionstherapie mit kristalloider Lösung wird weitergeführt. Bei einer metabolischen Azidose von max. 7,21 pH und einem Base Excess von $-9,6 \mathrm{mmol} / \mathrm{l}$ erfolgt eine Therapie mit Natriumbikarbonat $8,4 \%$. Eine Hyperkaliämie mit max. $6,2 \mathrm{mmol} / \mathrm{l}$ wird mit einer Actrapid-GlukoseInfusion sowie oben genannter Gabe von Natriumbikarbonat normalisiert.

Verlauf Im Verlauf bessert sich der Allgemeinzustand rasch. Der Methämoglobinspiegel sinkt ohne weitere Antidottherapie im Verlauf auf 1,2\% und der Patient wird am Folgetag auf die Normalstation verlegt. Der Verlauf dort ist unauffällig und der Patient kann einen Tag später nach Hause entlassen werden.

Methämoglobinanteil / Base Excess $\bigcirc$ Abb. 1 zeigt die Korrelation zwischen dem Methämoglobinanteil und dem Base Excess gegen den zeitlichen Verlauf (gemäß Uhrzeit). Innerhalb von 30 min nach Ankunft im Schockraum und der Verabreichung von Methylenblau sank der Methämoglobinanteil vom initialen Wert von 43,2\% auf $22,6 \%$. Im Verlauf kam es zu einem spontanen Anstieg des Methämoglobinanteils, jedoch normalisierte sich dieser ohne eine erneute Gabe von Methylenblau. Der Base Excess war initial nicht messbar, normalisierte sich aber im Verlauf ebenfalls.

- Bereits $18 \mathrm{~h}$ nach Eintritt befanden sich beide Werte wieder im Normbereich.

\section{Diskussion}

Verbreitung von „Poppers“ „Poppers“(volatile Alkylnitrite) sind seit Jahrzehnten in der Partyszene verbreitet. $\mathrm{Zu}$ den Alkylnitriten gehören neben Amylnitrit z.B. Butyl- und Isobutylnitrit [1]. Epidemiologische Informationen zu Verbreitung und Inzidenz sind sehr begrenzt, da sie oft unter die Kategorie des Missbrauchs mit inhalativen Substanzen fallen [2].

- Das Toxikologische Zentrum Zürich bekam im Zeitraum von 2009-2014 72 Anfragen [3];

- der Giftnotruf Berlin von 2009-2013 25 Anfragen [4].

- Die Vergiftungsinformationszentrale in Wien registrierte im Zeitraum 2002-2014 33 Anfragen zu Poppers, wobei in 17 Fällen systemische Symptome registriert wurden. In 4 Fällen handelte es sich um Mischintoxikationen, in 3 Fällen wurde Methylenblau empfohlen [5].

Wirkung Die farblose oder leicht gelbliche Flüssigkeit wird direkt aus der Flasche oder einem Tuch inhaliert und führt zu Euphorie, Wärmegefühl, Vasodilatation, vermindertem Schmerz- empfinden und zu einer Relaxation des Analsphinkters. Die Wirkung der volatilen Nitrite ist mit 1-2 min sehr kurz. Sie werden rasch durch Leber, Niere, Darmmukosa und Gefäßwand metabolisiert. Hier spielt die Glutathion-NitratReduktase in der Leber die Hauptrolle $[1,6]$ Die schnelle Metabolisierungsrate macht einen direkten Nachweis im Blut sehr schwierig.

Poppers gehören zu den 20 am häufigsten benutzten Drogen und stehen unter diesen jedoch bezüglich Gefährlichkeit an drittletzter Stelle [7]. Dennoch können, wie in unserem Fall, durch falsche Anwendung oder Überdosierung lebensgefährliche Komplikationen entstehen.

\section{Geschichtliches \\ $\nabla$}

Herkunft Amylnitrit wurde bereits 1859 für seine vasodilatatorischen Effekte bekannt. Durch die Inhalation der Substanz röten sich Gesicht und Hals [2]. Acht Jahre später wurde Amylnitrit in Anbetracht seiner vasodilatatorischen Eigenschaften für die Therapie von Angina pectoris eingesetzt [8]. Die Alkylnitrite waren initial in Glaskapseln verfügbar und deren Zerstörung ergab ein knallendes Geräusch.

- Dieses sog. „Popping“ führte wahrscheinlich zu dem englischen Namen „Poppers“ [2].

Falsche Annahmen In früheren Publikationen wurde vermutet, dass der Gebrauch volatiler Nitrite die Ursache zur Entwicklung von HIV und Kaposi-Sarkom sei. Es wurde sogar behauptet, dass eine Reduktion des Konsums von Alkylnitriten eine Verminderung der HIV-Epidemie bringen könnte [9]. Dies erwies sich später als falsch.

Abb. 1

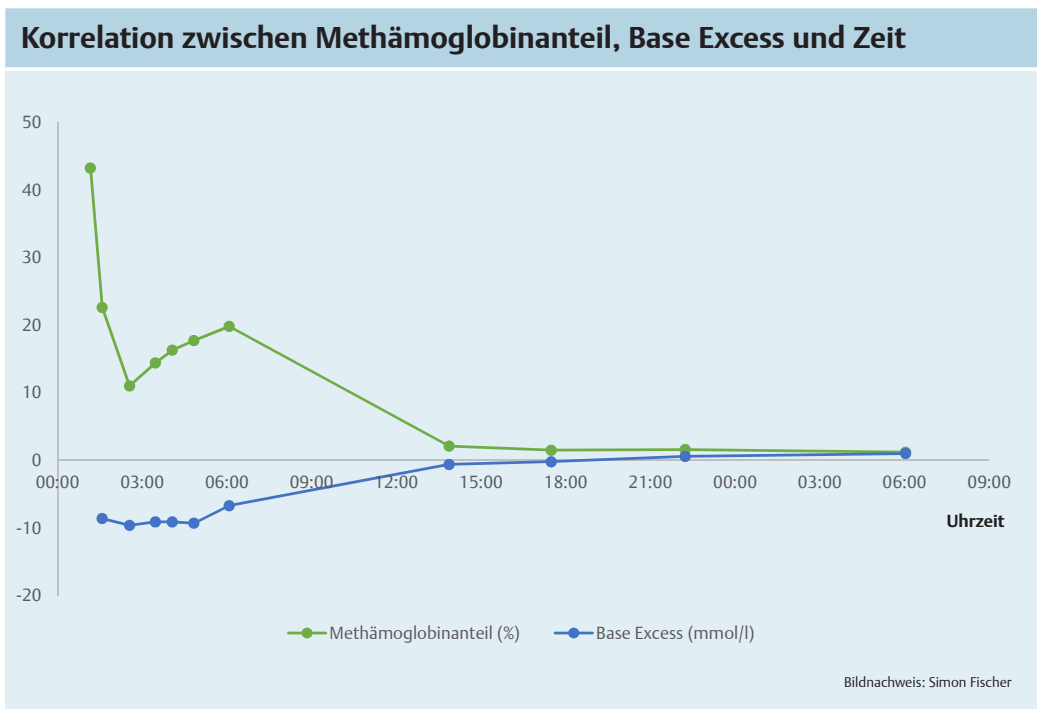


Tab. $1 \quad \mathrm{~F}_{\text {MetHb }}=$ Methämoglobin-Fraktion. Daten aus [2].

\begin{tabular}{|l|l|}
\hline \multicolumn{2}{|l|}{$\begin{array}{l}\text { Klinische Symptome abhängig vom } \\
\text { Ausmaß der Methämoglobinämie }\end{array}$} \\
\hline $\mathbf{F}_{\text {MetHb }}$ & Vergiftungssymptome \\
\hline$<15$ & oft keine Symptome \\
\hline $15-30$ & $\begin{array}{l}\text { Zyanose, Kopfschmerzen, } \\
\text { Schwindel }\end{array}$ \\
\hline $30-50$ & $\begin{array}{l}\text { Tachykardie, Dyspnoe, Schwäche, } \\
\text { Kollapsneigung, Lethargie, } \\
\text { Konfusion }\end{array}$ \\
\hline$>50$ & $\begin{array}{l}\text { schwere (Laktat-)Azidose, } \\
\text { Herzrhythmusstörungen, } \\
\text { Krampfanfälle }\end{array}$ \\
\hline$>70-80$ & potenziell letale Dosis \\
\hline
\end{tabular}

\section{Rechtliches und Vertrieb \\ $\nabla$}

Deutschland: Besitz und Konsum nicht strafbar Besitz und Konsum von Alkylnitriten unterliegen in diversen EU-Ländern nicht dem Betäubungsmittelgesetz und sind somit nicht strafbar. In den USA sowie in diversen EU-Ländern ist der Verkauf oder Handel von Alkylnitriten verboten, da sie dem Arzneimittelgesetz unterliegen und nitrathaltige Medikamente rezeptpflichtig sind. Sie können aber problemlos sehr günstig über Onlineshops bezogen werden. Die kleinen Fläschchen werden unter diversen Namen wie „Liquid Gold“, „Purple Haze“ und „Jungle Juice“ oder als Zimmerduft, Lederreinigungsmittel oder sonstige Putzmitteln getarnt $[1,10]$.

\section{Pathophysiologie}

Gefahr bei Ingestion Obwohl der Effekt der inhalierten Nitrite befristet ist, besteht eine große Gefahr bei der akzidentellen Ingestion. Einerseits kommt es zu einer Reduktion von Nitrit zu Stickstoffmonoxid (NO) [11]. Dessen vasodilatatorischer Effekt bewirkt verschiedene Symptome wie Flush, arterielle Hypotension, Reflextachykardie, Schwindel und Kopfschmerzen. Im extremen Fall kann ein manifester Schock entstehen. Andererseits kommt es zur raschen Entwicklung einer Methämoglobinämie. 2 Enzymsysteme, die zum Abbau von Methämoglobin führen. Daten aus [24]. MetHb $=$ Methämoglobin.

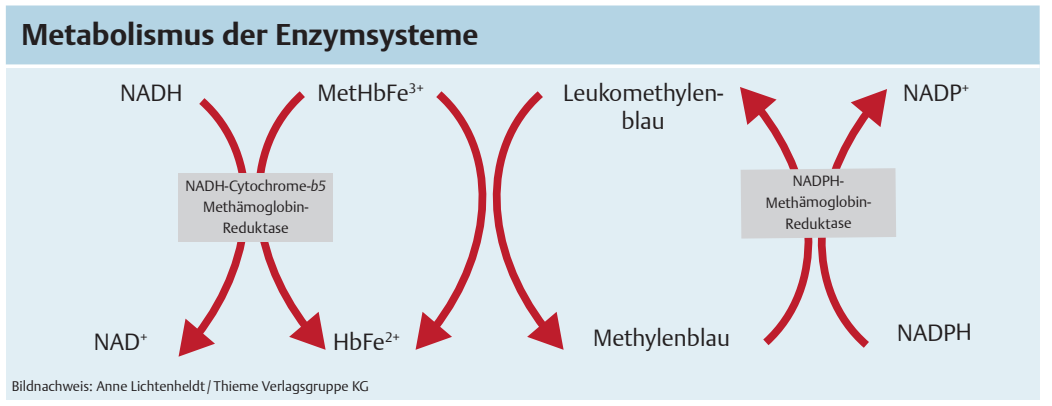

Auswirkungen Im Falle der Alkylnitritintoxikation kommt es zur übermäßigen Produktion von NO und Methämoglobin (MetHb). Der Körper bzw. seine Schutzmechanismen sind nicht mehr in der Lage, diese zu eliminieren [1]. Wie in unserem Fall beschrieben kommt es nach der oralen Ingestion zu einer schweren Vasodilatation mit der entsprechenden Hypotonie und Reflextachykardie sowie einer Hypoxämie. Der Schweregrad der Symptome korreliert dabei mit der Methämoglobinkonzentration ( $\bullet$ Tab. 1). Das MetHb kann einerseits kein $\mathrm{O}_{2}$-Molekül mehr binden und führt andererseits dazu, dass das noch vorhandene Hämoglobin eine höhere Affinität für $\mathrm{O}_{2}$ aufweist und somit zu einer Linksverschiebung der $\mathrm{O}_{2}$-Dissoziationskurve führt. Die $\mathrm{O}_{2}$-Abgabe in der Peripherie wird dadurch erschwert [13, 14].

Enzymsysteme Unter physiologischen Bedingungen hat der Körper 2 Enzymsysteme, die für die Elimination von MetHb sorgen. Diese führen dazu, dass die Konzentration von MetHb $<1 \%$ des gesamten Hämoglobins bleibt [12]. Hauptsächlich erfolgt die Reduktion des MetHb zurück zu Hämoglobin durch das Enzym NADH-Cytochromb5-Reduktase [13]. Wie $\odot$ Abb. 2 zeigt, reduziert dieses Enzym $\mathrm{Fe}^{3+} \mathrm{zu} \mathrm{Fe}^{2+}$, wobei das Letztere eine normale $\mathrm{O}_{2}$-Bindung und -Abgabe ermöglicht. Das zweite Enzymsystem ist das NADPH-MetHbReduktase-System. Es sorgt für nur 5\% der Elimination von MetHb, benutzt NADPH als Substrat und kann durch verschiedene externe Substanzen (z.B. Methylenblau) aktiviert bzw. gesteigert werden [14].

Andere Substanzen Neben den Alkylnitriten gibt es diverse andere Substanzen, die zu einer Methämoglobinbildung führen können. ๑ Tab. 2 zählt diese verschiedenen, zum Teil häufig benutzten Medikamente und Chemikalien auf [6].

\section{Pulsoxymetrie}

Falsche Beurteilung möglich Die Pulsoxymetrie gehört zu den wesentlichen Überwachungsverfahren jedes Patienten.

Im Fall einer Methämoglobinämie kann es jedoch rasch zu einem falschen Wert und damit auch zur falschen Beurteilung des Patienten kommen.

Der übliche Pulsoxymeter misst die Aufnahme von Oxy- und Deoxyhämoglobin $\left(\mathrm{O}_{2 \mathrm{Hb}}\right.$ und $\left.\mathrm{H}_{\mathrm{Hb}}\right)$ auf 2 verschiedenen Wellenlängen, nämlich 660 und $940 \mathrm{~nm}$. Unter normalen Bedingungen ergibt der Quotient zwischen der Aufnahme der roten $(660 \mathrm{~nm})$ und der infraroten Lichtwelle $(940 \mathrm{~nm})$ die $\mathrm{O}_{2}$-Sättigung. Bei einer Methämoglobinämie wird das MetHb praktisch in gleichen Anteilen von beiden Lichtwellen absorbiert, wodurch sich 
der Quotient an angleicht. Aufgrund der Kalibrierungskurve wird auf den meisten Geräten ein Quotient von 1 als eine $\mathrm{O}_{2}$-Sättigung von $85 \%$ definiert [15].

- Daher wird die peripher gemessene $\mathrm{O}_{2}$-Sättigung je nach Höhe des Methämoglobinspiegels über- oder unterschätzt. [16].

Neuerdings sind Geräte auf dem Markt, die eine nicht invasive Methämoglobinmessung erlauben.

\section{Diagnostik \\ $\nabla$}

Oxygen saturation gap Neben der Anamnese einer $\mathrm{O}_{2}$-refraktären Zyanose, der typisch schokoladebraunen Blutfarbe und dem direkten Methämoglobinnachweis im Blut kann der sog. „oxygen saturation gap" ein Hinweis für eine potenzielle Störung des Hämoglobins sein.

Definitionsgemäß muss hierfür die Differenz zwischen der $\mathrm{O}_{2}$-Sättigung im arteriellen Blut und der Pulsoxymetrie $>5 \%$ betragen $[17,18]$.

\section{Antidottherapie}

Methylenblau Methylenblau wurde im späten 19. Jahrhundert als Farbstoff für die Textilindustrie synthetisiert und praktisch gleichzeitig durch Robert Koch zur mikroskopischen Färbung von Tuberkulosebakterien verwendet. Obwohl sein Nutzen in der Textilindustrie nie wirklich an Bedeutung gewonnen hat, wurde Methylenblau schnell für diverse medizinische Indikationen eingesetzt [19], u.a. zur Therapie von Malaria, in der Psychiatrie, zur Therapie der Zyanid-Vergiftung und als Therapie bei Patienten mit Schock [19].

Methylenblau als Antidot Methylenblau als Antidot der Methämoglobinämie führt als Substrat des NADPH-MetHb-Reduktase-Systems zur Reduktion des MetHb. Die übliche Dosierung von Methylenblau beträgt $1-2 \mathrm{mg} / \mathrm{kg}$. Bei einer Dosierung von $>4 \mathrm{mg} / \mathrm{kg}$ kann es zur Verfärbung des Stuhls, der Haut und des Urins kommen. In der Regel kommt es nach 30 min zu einem Abfall des Methämoglobinspiegels. Methylenblau in Dosierungen $>7 \mathrm{mg} / \mathrm{kg}$ kann zur paradoxen Methämoglobinämie führen $[19,20]$.

Glukose-Dehydrogenase-Mangel Bei einem Therapieversagen von Methylenblau oder einer nach Methylenblau induzierten Hämolyse muss an einen Glukose-6-Dehydrogenase-Mangel gedacht werden, da die Glukose-6-Dehydrogenase Voraussetzung für die Entstehung von NADPH ist.

\begin{tabular}{|c|c|c|}
\hline \multicolumn{3}{|c|}{ Alltägliche Substanzen, die zur Methämoglobinämie führen können } \\
\hline $\begin{array}{l}\text { Paracetamol } \\
\text { - Acetanilid } \\
\text { - Aminophenole } \\
\text { - Ammoniumnitrat } \\
\text { - Amylnitrat } \\
\text { - Anilin } \\
\text { - Celecoxib } \\
\text { - Chloroquin } \\
\text { - Kobalt } \\
\text { - Chlorate } \\
\text { - Kupfersulfat } \\
\text { Dapson } \\
\text { EMLA } \\
\text { - Herbizide }\end{array}$ & $\begin{array}{l}\text { Hydroxylamin } \\
\text { - Lokalanästhetika } \\
\text { - Benzocain } \\
\text { - Kokain } \\
\text { - Lidocain } \\
\text { - Prilocain } \\
\text { - Procain } \\
\text { - Menthol } \\
\text { - Methylenblau } \\
\text { - Nitrat/ Nitrit } \\
\text { - Nitrofurantoin } \\
\text { - Stickstoffoxid } \\
\text { - Nitroglyzerin } \\
\text { - Nitroprussid }\end{array}$ & $\begin{array}{l}\text { Nitrosobenzol } \\
\text { Phenacetin } \\
\text { Phenole } \\
\text { - Phenytoin } \\
\text { - Primaquin } \\
\text { - Pyridin } \\
\text { - Pyridium } \\
\text { - Chinon } \\
\text { - Resorcinol } \\
\text { - Silbernitrat } \\
\text { Sodiumnitrit } \\
\text { Sulfonamid } \\
\text { P Trinitrotoluen }\end{array}$ \\
\hline
\end{tabular}

Tab. 2

Der Glukose-6-Dehydrogenasemangel wird Xchromosomal-rezessiv vererbt und ist im Mittelmeerraum häufig verbreitet. In diesem Falle kann eine Austauschtransfusion lebensrettend sein [21].

\section{Andere Nebenwirkungen \\ $\nabla$}

Visusstörung/Makulopathie Kreislaufkollaps und Methämoglobinämie sind nicht die einzigen Gefahren, die mit Poppers verbunden sind. Davies et al. haben vor kurzem eine Fallserie über Poppers-induzierte Visusstörung und Makulopathie publiziert. Interessanterweise hatten nur 2 von den 7 Patienten Poppers regelmäßig inhaliert. Alle anderen Patienten waren bereits nach dem ersten Mal symptomatisch [10].

Kontaktdermatitis Schauber und Herzinger beschreiben 2 Fälle von Kontaktdermatitis, die durch einen stundenlangen Hautkontakt mit Poppers verursacht wurden [22].

Betroffene Kinder Wie es bei Intoxikationen häufig der Fall ist, können auch Kinder betroffen sein. Die jüngste Patientin ist ein 2-jähriges Mädchen, das schätzungsweise $5 \mathrm{ml}$ „Liquid Gold“ akzidentell aus einer zu Hause gefundener Flasche eingenommen hat [23].

Fazit Alkylnitrite sind heute als euphorisierende und muskelrelaxierende Partydroge bekannt. Da sie häufig zum Analverkehr eingesetzt wird, ist sie in homosexuellen Kreisen weit verbreitet. Sie wird direkt inhaliert und kann bei Ingestion schwerwiegende Nebenwirkungen wie Vasodilatation und Methämoglobinbildung hervorrufen. Die Diagnose kann durch die Anamnese einer $\mathrm{O}_{2}$-refraktären Zyanose, der typisch schokoladebraunen Blutfarbe und der Bestimmung von MetHb gestellt werden. Die Therapie besteht aus der Antidottherapie mit Methylenblau und kreislaufunterstützenden Maßnahmen. 4 


\section{Kernaussagen \\ Die orale Einnahme von Poppers kann letale Konsequenzen haben. \\ Dosisabhängig kommt es zur Methämoglobin- bildung.}

Charakteristisch sind eine $\mathrm{O}_{2}$-refraktäre Zyanose und die schokoladebraune Blutfarbe.

Die Diagnose wird laborchemisch durch den Nachweis von MetHb gestellt.

Die pulsoxymetrisch gemessene $\mathrm{O}_{2}$-Sättigung kann je nach Höhe des Methämoglobinspiegels falsch hohe oder falsch tiefe Werte anzeigen.

Die Antidottherapie besteht aus der i. v. Gabe von Methylenblau (1-2 mg/kg KG).

Cave: Ein Glukose-6-Dehydrogenase-Mangel kann eine Hämolyse auslösen.

Eine Rücksprache mit dem toxikologischen Zentrum wird empfohlen.

Beitrag online zu finden unter http://dx.doi. org/10.1055/s-0040-100107

\section{Literaturverzeichnis}

1 Romanelli F, Smith KM, Thornton AC, Pomeroy C. Poppers: Epidemiology and clinical management of inhaled nitrite abuse. Pharmacotherapy 2004; 24: 69-78

2 Newell GR, Mansell PW, Spitz MR et al. Volatile nitrites. Use and adverse effects related to the current epidemic of the acquired immune deficiency syndrome. Am J Med 1985; 78: 811-816

3 Degrandi C. Schweizerisches Toxikologisches Informationszentrum Zürich. Persönliche Kommunikation 19.09.2014

4 Acquarone D. Giftnotruf der Charité - Universitätsmedizin Berlin. Persönliche Kommunikation 19.09.2014.

5 Schiel H, Genser D. Vergiftungsinformationszentrale Wien. Persönliche Kommunikation 12.09.2014.

6 Sheena Y, Baston EL, Downs A, Chester DL. A sticky situation: methaemaglobinaemia in a hand trauma patient. BMJ Case Rep 2012. pii: bcr2012007196

7 Nutt D, King LA, Saulsbury W, Blakemore C. Development of a rational scale to assess the harm of drugs of potential misuse. Lancet 2007; 369: 1047-1053

8 Brunton TL. On the use of nitrite of amyl in angina pectoris. Lancet 1867; 2: 97-98

9 Haverkos HW, Pinsky PF, Drotman DP, Bregman DJ. Disease manifestation among homosexual men with acquired immunodeficiency syndrome: a possible role of nitrites in Kaposi's Sarcoma. Sex Transm Dis 1985; 12: 203-208

10 Davies AJ, Kelly SP, Naylor SG et al. Adverse ophthalmic reaction in poppers users: case series of ,poppers maculopathy'. Eye 2012; 26: 1479-1486

11 Lundberg JO, Weitzberg E, Gladwin MT. The nitrate-nitritenitric oxide pathway in phsysiology and therapeutics. Nat Rev Drug Discov 2008; 7: 156-167

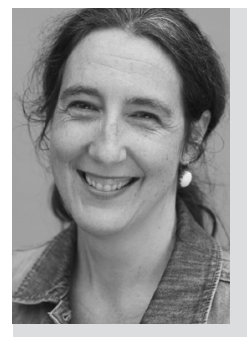

Dr. med. Barbara Bernasconi ist Oberärztin an der Klinik für Anästhesie, Intensivmedizin, Rettungsmedizin und Schmerztherapie des Luzerner Kantonsspitals. Sie ist Mitglied der Fédération des médecins suisses (FMH) und der Schweizerischen Gesellschaft für Notfall- und Rettungsmedizin.

E-Mail: barbara.bernasconi@luks.ch

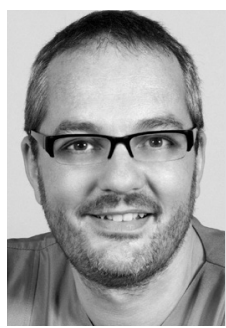

Prof. Dr. med. Christoph Konrad ist Chefarzt an der Klinik für Anästhesie, Intensivmedizin, Rettungsmedizin und Schmerztherapie des Luzerner Kantonsspitals.

E-Mail: christoph.konrad@luks.ch

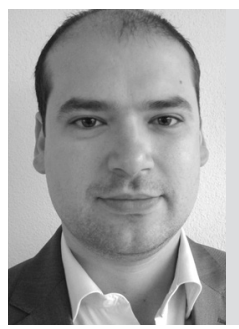

Dr. med. Simon Fischer, PhD, ist Assistenzarzt an der Klinik für Anästhesie, Intensivmedizin, Rettungsmedizin und Schmerztherapie des Luzerner Kantonsspitals. E-Mail: simon.fischer@luks.ch

Interessenkonflikt Die Autoren erklären, dass keine Interessenkonflikte vorliegen.

12 Wright RO, Lewander WJ, Woolf AD. Methemoglobinemia: etiology, pharmacology, and clinical management. Ann Emerg Med 1999; 34: 646-656

13 Mansouri A, Lurie AA. Concise review: methemoglobinemia. Am J Hematol 1993; 42: 7-12

14 Curry S. Methemoglobinemia. Ann Emerg Med 1982; 11; 214-221

15 Haymond S, Cariappa R, Eby CS, Scott MG. Laboratory assessment of oxygenation in methemoglobinemia. Clin Chem 2005; 51: 434-444

16 Zijlstra WG, Buursma A, Meeuwsen-van der Roest WP. Absorption spectra of human fetal and adult oxyhemoglobin, de-oxyhemoglobin, carboxyhemoglobin, and methemoglobin. Clin Chem 1991; 37: 1633-1638

17 Akhtar J, Johnston BD, Krenzelok EP. Mind the gap. J Emerg Med 2007; 33: 131-132

18 Toffaletti J, Zijlstra WG. Miscocenptions in reporting oxygen saturation. Anesth Analg 2007; 105 (Suppl. 6): S5-9

19 Lo JCY, Darracq MA, Clark RF. A review of methylene blue treatment for cardiovascular collapse. J Emerg Med 2014; 46: 670-679

20 Hall A, Stessel B, Bergmans D, Schnabel R. Two cases of acquired methemoglobinemia. Acta Anaesth Belg 2013; 63: 97-100

21 Wellershoff G. Potenziell letale Methämoglobinämie nach Ingestion von Alkylnitriten („Poppers“). Notfall Rettungsmed 2014; 17: 137-140

22 Schauber J, Herzinger T. ,Poppers' Dermatitis. Clin Exp Dermatol 2012; 37: 587-588

23 Forsyth RJ, Moulden A. Methaemoglobinaemia after ingestion of amyl nitrite. Arch Dis Child 1991; 66: 152

24 Wilkerson RG. Getting the blues at a rock concert: a case of severe methaemoglobinaemia. Emerg med Australas 2010; 22: 466-469 